\title{
Über quantitative Bestimmung der im Proteine oder in dessen Abbauprodukten vorhandenen Peptidbindungen.
}

\author{
Von
}

V. Henriques und J. K. Gjaldbäk.

(Aus dem physiologischen Laboratorium der königl. tierärztlichen und landwirtschaftlichen Hochschule Kopenhagen.)

(Der Redaktion zugegangen am 7. Mai 1910.)

In einer früheren Abhandlung teilte Henriques ${ }^{1}$ ) eine Reihe von Versuchen über die Menge peptidgebundenen Stickstoffes in mehreren verschiedenen Stoffen mit, die teils durch Verdauung mit Trypsin-Erepsin, teils durch Erwärmung auf $100^{\circ}$ (auf dem Wasserbade) mit 20-30\% iger Schwefelsäure aus Proteinen dargestellt worden waren. Was das angewandte Verfahren betrifft, verweisen wir auf die obengenannte $A b-$ handlung; hier rufen wir nur ins Gedächtnis zurück, daß das Verfahren in Kürze folgendes bezweckte: In einer Lösung des zur Untersuchung vorliegenden Stoffes bestimmte man nach genauer Neutralisation mit Lackmus mittels der Formoltitrierung S. P. L. Sörensens die Menge der vorhandenen freien $\mathrm{NH}_{2}-$ Gruppen. Ein anderer Teil der Lösung wurde $2 \mathrm{mal} \mathrm{mit} \mathrm{starker}$ Salzsäure auf dem Wasserbade eingedampft; den Rest löste man in Wasser auf, neutralisierte mit Lackmus als Indikator, worauf man formoltitrierte. Die Differenz zwischen den beiden gefundenen Mengen titrierbarer Carboxylgruppen liefert dann ein Maß für die im untersuchten Stoffe befindliche Menge peptidgebundenen Stickstoffes. ${ }^{2}$ ) Es versteht sich von selbst, daß

1) V. Henriques, Die Eiweißsynthese im tierischen Organismus. Diese Zeitschrift, Bd. LIV.

2) Was die näheren Verhältnisse der Formoltitrierung betrifft, verweisen wir ferner auf: S. P. L. Sörensen, Enzymstudien, Biochem. Zeitschrift, Bd. VII, und auf S. P. L. Sörensen und H. Jessen-Hansen, ibid., Bd. VII. 
eine der Bedingungen für die Richtigkeit des Verfahrens die sein muß, daß man imstande ist, durch Eindampfen mit konzentrierter Salzsäure auf dem Wasserbade den vollständigen Abbau des Proteins und der Spaltungsprodukte desselben zu erzielen; ist dies nicht der Fall - enthält der mit Salzsäure behandelte Stoff noch andere Peptidbindungen, die sich durch weitere Behandlung mit Säure lösen lassen -, so werden wir einen Fehler dadurch begehen, daß der supponierte "Nullpunkt» nicht richtig bestimmt worden ist.

Um dieses Verhalten näher zu untersuchen und um womöglich ein Verfahren zur sicheren Bestimmung des Gehalts der Proteinstoffe und ihrer Spaltungsprodukte an peptidgebundenem Stickstoffe zu finden, haben wir eine Reihe von Untersuchungen angestellt, die wir hier näher erörtern werden.

Was das von uns angewandte Verfahren betrifft, so wird folgendes Beispiel dasselbe leicht verständlich machen. Um die Menge peptidgebundenen Stickstoffes in Witte-Pepton zu untersuchen, gingen wir von einer ca. 4\% igen Lösung aus, die nach der Kjeldahl-Analyse in $5 \mathrm{ccm}$ einen Stickstoffgehalt von $57,25 \mathrm{mg}$ zeigte.

$25 \mathrm{ccm}$ der Löșung verdünnten wir in einem Meßkolben bis auf $200 \mathrm{ccm}$, nachdem wir vorher mit Lackmuspapier möglichst genau neutralisiert hatten; von dieser Lösung nahmen wir $40 \mathrm{ccm}$ zur Formoltitrierung, während wir andere $40 \mathrm{ccm}$ zur Ammoniakbestimmung anwandten (die stets durch Destillation im Vakuum mit Baryt, in Methylalkohol gelöst, ausgeführt wurde). Die Formoltitrierung ergab 13,44 mg formoltitrierbaren Stickstoffes und die Ammoniakbestimmung $0,80 \mathrm{mg}$ Stickstoff als Ammoniak; ${ }^{1}$ ) die Menge des Aminosäurestickstoffes wird mithin: $13,44 \div 0,80=12,64 \mathrm{mg}$.

$25 \mathrm{ccm}$ der ursprünglichen Peptonlösung brachten wir in eine Porzellanschale, wir setzten $25 \mathrm{ccm}$ konzentrierte Salzsäure zu und dampften die Flüssigkeit bis zur Trockne ein, setzten von neuem Salzsäure zu und dampften wieder bis zur Trockne ein. Darauf brachten wir den Rest mit Hilfe von

1) Über das Verhalten des Ammoniaks siehe näheres unten. 
Wasser in einen $100 \mathrm{ccm}-M e ß k o l b e n$. Die Flüssigkeit ist gewöhnlich stark braunfarbig; man entfärbt durch Fällung mit $\left.\mathrm{AgNO}_{3}(\mathrm{n} / \mathrm{s})^{1}\right)$ und verdünnt bis zur Marke $(+0,2 \mathrm{ccm}$ für das gefällte Silberchlorid). Von dem jetzt nur schwach gelblichen Filtrate führt man $50 \mathrm{ccm}$ in einen $100 \mathrm{ccm}-K o l b e n$ über, man neutralisiert die Flüssigkeit genau mit Lackmuspapier und verdünnt bis zur Marke. Von dem Inhalt des Kolbens nimmt man $40 \mathrm{ccm}$ ( $=5 \mathrm{ccm}$ der ursprünglichen Lösung) zur Formoltitrierung und $40 \mathrm{ccm}$ zur Ammoniakbestimmung. Hierdurch fanden wir $36,80 \mathrm{mg}$ formoltitrierbaren Stickstoff und 4,05 mg Ammoniakstickstoff.

Von der ursprünglichen Witte-Peptonlösung nahmen wir ferner 3 , je $25 \mathrm{ccm}$ große Proben; wir setzten $25 \mathrm{ccm}$ konzentrierte Salzsäure zu jeder Probe und kochten darauf die Mischungen über freier Flamme 1 bezw. 3 und 12 Stunden lang mit Rückflußkühler. Nach beendigtem Kochen entfernten wir die Salzsäure durch Eindampfen auf dem Wasserbade, und darauf ist das Verfahren ganz das oben beschriebene. Das Resultat des Versuches war nun folgendes:

Witte-Peptonlösung. Totaler $\mathrm{N}$ in $5 \mathrm{ccm}=57,25 \mathrm{mg}$.

\begin{tabular}{c|c|c|c}
\hline \hline & $\begin{array}{c}\text { Formoltitrierter N } \\
\mathrm{mg}\end{array}$ & $\begin{array}{c}\text { Ammoniak-N } \\
\mathrm{mg}\end{array}$ & $\begin{array}{c}\text { Aminosäure-N } \\
\mathrm{mg}\end{array}$ \\
\hline Sogleich bestimmt . . & 13,44 & 0,80 & 12,64 \\
Zweimal mit HCl auf dem & 36,80 & 4,05 & 32,75 \\
Wasserbade eingedampft & 41,72 & 3,95 & 37,77 \\
1 Stunde mit HCl gekocht & 43,68 & 3,95 & 39,73 \\
3 Stunden > . > & 44,94 & 4,40 & $\mathbf{4 0 , 5 4}$
\end{tabular}

Aus den gefundenen Zahlen ist zu ersehen, daß das Witte-Pepton durch zweimaliges Eindampfen auf dem Wasserbade mit konzentrierter $\mathrm{HCl}$ nicht vollständig abgebaut wird, indem nämlich die Formoltitrierung im Verein mit der Ammoniakbestimmung einen Aminosäurestickstoffgehalt von $32,75 \mathrm{mg}$ ergab, während 12 stündiges Kochen mit ca. 20\% iger HCl die Menge des Aminosäurestickstoffes bis auf $40,54 \mathrm{mg}$ brachte. Ferner

1) Siehe S. P. L. Sörensen und H. Jessen-Hansen, l. c. 
sieht man, daß weder 1 - noch 3 stündiges Kochen mit $\mathrm{HCl}$ imstande war, den völligen Abbau des Peptons zu bewirken. Inwiefern wir durch 12 stündiges Kochen mit $\mathrm{HCl}$ den Abbau zu Ende gebracht haben, läßt sich aus den in diesem Versuche gefundenen Zahlen nicht ermitteln; wie wir indes unten sehen werden, hat sich die Hydrolyse nach 12 stündigem Kochen wirklich vollzogen oder gibt - mit anderen Worten - in dem angeführten Versuche die Zahl 40,54 den «Nullpunkt» an, von welchem aus wir den Grad des durch Einwirkungen, die das Pepton nicht vollständig spalten, hervorgebrachten Abbaus einzuschätzen vermögen.

Die natürlichste Weise, den Spaltungsgrad auszudrücken, ist die, daß man die Menge des noch ferner lösbaren peptidgebundenen Stickstoffes in Prozenten der vorhandenen Menge Aminosäurestickstoffes angibt; im obigen Versuche war z. B. die Gesamtmenge Aminosäurestickstoffes $=40,54 \mathrm{mg}$; durch zweimaliges Eindampfen auf dem Wasserbade mit $\mathrm{HCl}$ fand man $32,75 \mathrm{mg} \mathrm{N}$; nach dem Eindampfen auf dem Wasserbade waren mithin noch $40,54 \div 32,75=7,79 \mathrm{mg}$ peptidgebundenen Stickstoffes übrig, was $19,2 \%$ der $40,54 \mathrm{mg} \mathrm{N}$ beträgt, oder, mit anderen Worten, es finden sich in dem mit Salzsäure auf dem Wasserbade behandelten Stoffe noch 19,2\% peptidgebundenen Stickstoffes.

In einigen Fällen verwandten wir, wie aus untenstehenden Versuchen zu ersehen, Schwefelsäure statt Salzsäure zur Hydrolyse. Sonst war das Verfahren ganz das oben angegebene, nur setzten wir vor der Entfärbung mit $\mathrm{AgNO}_{3}$ eine geeignete Menge Natriumchloridlösung $\mathrm{zu}$.

Was die Genauigkeit des Verfahrens betrifft, so muß man wegen der vielen verschiedenen Manipulationen mit einem Fehler von ca. $2 / 10 \mathrm{ccm}$ bei der Titrierung rechnen, was wieder einen Fehler der Stickstoffmenge von $0,2 \times 2,8=0,56 \mathrm{mg}$ bezeichnet.

Was die in der Tabelle I angeführten Stoffe betrifft, so handelt es sich erstens um 4 Proben trypsin-erepsin-verdautes Kalbfleisch, das ca. 3-4 Monate im Thermostaten gestanden hatte; ferner finden sich Proben von Witte-Pepton, das teils unverändert, teils nach kürzerem oder längerem Stehen im 


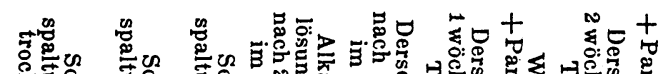

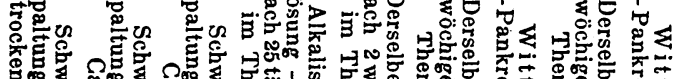
๑.

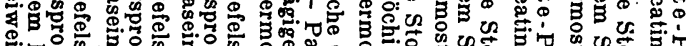

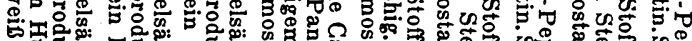

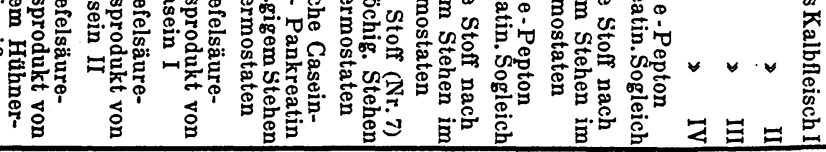

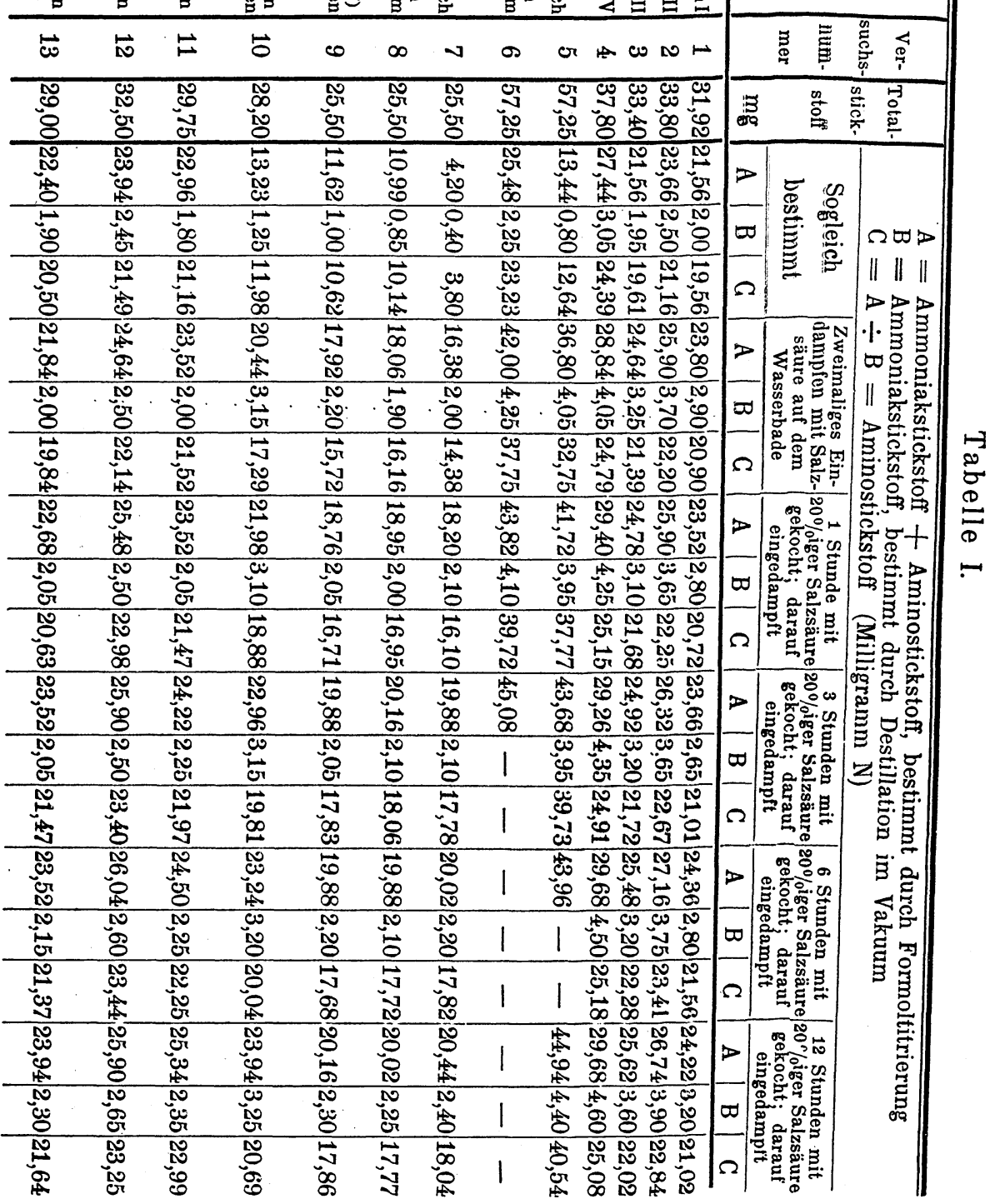


Thermostaten mit Pankreatin (Rhenania) untersucht wurde. Endlich untersuchten wir einige Spaltungsprodukte des Caseins (Hammarsten) und Hühnereiweißes (gewöhnliche Handels ware) durch ca. 15 stündiges Kochen einer größeren Menge der genannten Stoffe mit $25 \%$ iger Schwefelsäure über freier Flamme dargestellt.

Wie aus den angeführten Zahlen ersichtlich, genügt es nicht, die untersuchten Stoffe zweimal mit konzentrierter $\mathrm{HCl}$ auf dem Wasserbade einzudampfen, wenn man eine totale Hydrolyse erzeugen will, indem man überall eine nicht so gar wenig höhere Zahl durch die Formoltitrierung findet, nachdem der Stoff 12 Stunden lang mit konzentrierter $\mathrm{HCl}$ gekocht worden ist. Sogar in denjenigen Fällen, wo das Ausgangsmaterial ein Stoff war, von dem sich annehmen ließ, er sei sozusagen vollständig abgebaut, erweist es sich, daß zweimaliges Eindampfen auf dem Wasserbade mit Salzsäure nicht imstande war, die totale Hydrolyse hervorzurufen. Im Versuche Nr. 11, wo mit Schwefelsäure gespaltenes Casein angewandt wurde, fand man sogleich durch die Formoltitrierung $21,16 \mathrm{mg} \mathrm{N}$, nach $\mathrm{HCl}-$ Behandlung auf dem Wasserbade 21,52 mg. Nach 12 stündigem Kochen über freier Flamme fand man jedoch $22,99 \mathrm{mg} \mathrm{N}$, was besagt, daß der Stoff nach zweimaliger Behandlung auf dem Wasserbade mit $\mathrm{HCl}$ noch immer $6,4 \%$ peptidgebundenen Stickstoffs enthielt. Im Versuche Nr. 12, wo ebenfalls mit $\mathrm{H}_{2} \mathrm{SO}_{4}$ gespaltenes Casein zur Verwendung kam, findet man nach zweimaligem Eindampfen mit $\mathrm{HCl}$ noch $4,7 \%$ peptidgebundenen Stickstoffes. Im Versuch 13 wurde ein Präparat angewandt, das durch 15 stündiges Kochen mit $\mathrm{H}_{2} \mathrm{SO}_{4}$ aus gespaltenem Hühnereiweiß dargestellt worden war; hier findet sich sogar nach einstündigem Kochen mit $\mathrm{HCl}$ über freier Flamme noch 4,7\% peptidgebundenen Stickstoffs, welche Zahl übrigens etwas zu niedrig ist, indem Hühnereiweiß im Gegensatz zum Witte-Pepton und Casein nicht vollständig hydrolysiert wird, selbst wenn man es 12 Stunden lang mit konzentrierter $\mathrm{HCl}$ kocht; auf dieses Verhalten werden wir unten näher zurückkommen.

Betrachtet man die Zahlen für trypsin-erepsin-verdautes Fleisch, so sieht man, daß zweimaliges Eindampfen mit $\mathrm{HCl}$ 
auf dem Wasserbade stark auf diese Stoffe einwirkt, indem man nach dieser Behandlung in den vier angeführten Versuchen (Nr. 1, 2, 3 und 4) nur folgende Mengen peptidgebundenen Stickstoffes findet: $0,5 \%, 2,8 \%, 2,9 \%$ und 1,2\%. Da alle Versuche dartun, daß die Menge titrierbaren Stickstoffes nach 12stündigem Kochen mit Salzsäure größer ist als nach zweimaligem Eindampfen auf dem Wasserbade mit Salzsäure, hat man wahrscheinlich anzunehmen, daß die Hydrolyse nach dem Eindampfen auf dem Wasserbade nicht ganz beendigt war; übrigens sind die angeführten Zahlen so klein, daß man sie wohl zunächst als innerhalb der Fehlergrenze liegend betrachten darf.

Den größten Unterschied zwischen der Wirkung des zweimaligen Eindampfens auf dem Wasserbade und der des zwölfstündigen Kochens über freier Flamme mit Salzsäure findet man in denjenigen Fällen, wo das Ausgangsmaterial mehr kompliziert gebaute Stoffe waren, wie z. B. Witte-Pepton oder das einige Zeit hindurch mit Trypsin verdaute Casein. Im Versuche 5 wurde Witte-Pepton angewandt, das nach zweimaligem Eindampfen mit $\mathrm{HCl}$ bei Formoltitrierung $32,75 \mathrm{mg} \mathrm{N}$ gab, während die Titrierung nach 12 stündigem Kochen mit $\mathrm{HCl} 40,54 \mathrm{mg}$ zeigte; nach dem Eindampfen auf dem Wasserbade fanden sich also noch $19,2 \%$ peptidgebundenen Stickstoffes. Im Versuche Nr. 6 wurde dieselbe Peptonlösung benutzt, nachdem die Lösung jedoch 2 Wochen lang mit Trypsin im Thermostaten gestanden hatte. In diesem Falle ist die Menge des peptidgebundenen Stickstoffes nach zweimaligem Eindampfen auf dem Wasserbade mit Salzsäure bis auf $6,9 \%$ gesunken.

In den Versuchen 7, 8 und 9 kam ebenfalls Witte-Peptonlösung zur Verwendung, teils unverändert, teils nach Verdauung mit Pankreatin. Im ersteren Falle zeigt zweimaliges Eindampfen mit HCl auf dem Wasserbade einen Gehalt von 20,3\% peptidgebundenen Stickstoffs; nach der Pankreatinwirkung ist die Menge bis auf $(10,4$ bzw. 12,9) ca. $11,7 \%$ gesunken.

Endlich erweist eine Caseinlösung, die 25 Tage hindurch mit Pankreatin im Thermostaten gestanden hat, nach zweimaligem Eindampfen mit HCl auf dem Wasserbade einen Gehalt an peptidgebundenem Stickstoff von 16,4\%. 
Mit Bezug auf die Frage, wie lange das Kochen mit Salzsäure über freier Flamme dauern muß, um totale Hydrolyse zu erzeugen, erweist es sich, daß in denjenigen Fällen, wo das Ausgangsmaterial vorher stark hydrolysiert ist, ein sechsstündiges Kochen wohl stets genügen wird; handelt es sich dagegen um einen Stoff wie Witte-Pepton (Versuch Nr. 5), so muß man, um ganz sicher zu gehen, das Kochen mehr als 6 Stunden lang fortsetzen - wie lange, das läßt sich nach den in der Tabelle angegebenen Zahlen nicht entscheiden.

Da es sich erwies, daß die vollständige Hydrolyse der Proteine ein wenigstens 6 stündiges Kochen über freier Flamme mit HCl erforderte, versuchten wir, ob es nicht tunlich sei, die Hydrolyse auf andere Weise schneller zu vollführen; aus den unten angeführten Versuchen wird man sehen, daß $1^{1 / 2}$ stündige Erhitzung der Proteine auf $150^{\circ}$ mit Säure im Autoklaven in allen Fällen zum Ziele zu führen scheint. Wir wandten teils Schwefelsäure, teils Salzsäure in verschiedenen Konzentrationen an. Die Säuremenge ist in normaler Säure angegeben, und die von uns benutzten Säuremengen variierten zwischen 1- und 5-normal. Des Vergleiches wegen bestimmten wir gleichzeitig den Hydrolysegrad nach Kochen mit konzentrierter (20\%iger) HCl über freier Flamme 3, 6 und 12 Stunden hindurch.

\section{Tabelle II.}

Standardlösung $=$ ca. $4 \%$ ige Lösung von trockenem Hühnereiweiß.

$$
\begin{aligned}
& \mathrm{A}=\text { Ammoniakstickstoff }+ \text { Aminostickstoff } \\
& \mathrm{B}=\text { Ammoniakstickstoff } \\
& \mathrm{C}=\text { Aminostickstoff }(\mathrm{A} \div \mathrm{B}) \\
& \text { Totalstickstoff in } 5 \mathrm{ccm}=23,10 \mathrm{mg} .
\end{aligned}
$$

\begin{tabular}{|c|c|c|c|}
\hline & $\mathbf{A}$ & B & C \\
\hline Bestimmung sogleich ......... & 1,40 & 0,40 & 1,00 \\
\hline $\begin{array}{c}3 \text { Stunden mit } 20 \% \text { iger Salzsäure gekocht, } \\
\text { darauf eingedampft }\end{array}$ & 16,90 & 1,70 & 15,20 \\
\hline 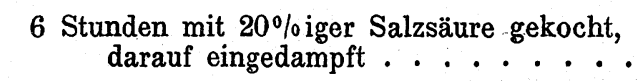 & 17,50 & 1,85 & 15,65 \\
\hline $\begin{array}{l}12 \text { Stunden mit } 20 \% \text { iger Salzsäure gekocht, } \\
\text { darauf eingedampft . . . . . . }\end{array}$ & 17,64 & 2,00 & 15,64 \\
\hline
\end{tabular}


Spaltung durch Erhitzung im Autoklaven.

\begin{tabular}{|c|c|c|c|c|c|c|c|c|c|c|}
\hline \multirow{2}{*}{\multicolumn{2}{|c|}{$\begin{array}{c}\text { Konzentration } \\
\text { und Art der } \\
\text { angewandten Säure }\end{array}$}} & \multicolumn{3}{|c|}{$\begin{array}{c}150^{\circ} \\
1^{1 / 2} \text { Stunden }\end{array}$} & \multicolumn{3}{|c|}{$\begin{array}{c}150^{\circ} \\
3 \text { Stunden }\end{array}$} & \multicolumn{3}{|c|}{$\begin{array}{l}180^{\circ} \\
11 / 2 \text { Stunden }\end{array}$} \\
\hline & & A & $\mathrm{B}$ & $\mathrm{C}$ & $\mathrm{A}$ & $\mathrm{B}$ & $\mathrm{C}$ & A & B & G \\
\hline \multicolumn{2}{|c|}{ 1-normal-Salzsäure . . } & 18,20 & 2,30 & 15,90 & 18,06 & 2,50 & 15,56 & 19,04 & 3,60 & 15,44 \\
\hline 2 & $»$ & 19,04 & 2,30 & 16,74 & 19,18 & 2,55 & 16,63 & 19,60 & 3,40 & 16,20 \\
\hline 3 & $\triangleright$ & 19,74 & 2,40 & 17,34 & 19,46 & 2,30 & 17,16 & 19,74 & 4,00 & 15,74 \\
\hline$t$ & 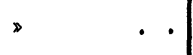 & 19,60 & 2,50 & 17,10 & 19,60 & 2,65 & 16,95 & 19,60 & 3,80 & 15,80 \\
\hline & $\triangleright$ & 19,60 & 2,65 & 16,95 & 19,60 & 2,85 & 16,75 & 20,02 & 3,80 & 16,22 \\
\hline \multicolumn{2}{|c|}{ 1-normal-Schwefelsäure } & 17,08 & 2,40 & 14,68 & - & - & - & 18,34 & 3,80 & 14,54 \\
\hline$?$ & $\triangleright$ & 18,62 & 2,60 & 16,02 & 18,76 & 2,80 & 15,96 & 19,46 & 3,80 & 15,66 \\
\hline & 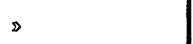 & 18,90 & 2,70 & 16,20 & 19,04 & 2,90 & 16,14 & 19,60 & 4,00 & 15,60 \\
\hline 4 & $\gg$ & 18,90 & 2,65 & 16,25 & 19,32 & 2,85 & 16,47 & 19,60 & 4,30 & 15,30 \\
\hline D & D & 19,32 & 2,60 & 16,72 & - & - & - & 19,47 & 4,40 & 15,34 \\
\hline
\end{tabular}

Die angeführte Versuchsreihe wurde mit einer ca. $4 \%$ igen Lösung von gewöhnlichem, gedörrtem Hühnereiweiß ausgeführt. Es zeigt sich, daß 12 stündiges Kochen mit Salzsäure über freier Flamme keine stärkere Hydrolyse als 6 stündiges Kochen gibt. Dies sollte zu dem Schluß berechtigen, daß die Hydrolyse nach 6 stündigem Kochen eine vollständige wäre. Indes sieht man, daß $11 / 2$ stündige Erhitzung auf $150^{\circ}$ mit einer Säuremenge $=3-\mathrm{n}-\mathrm{HCl} \mathrm{im}$ Autoklaven ein nicht geringes Steigen des Hydrolysegrades bewirkt. Nach Kochen über freier Flamme fand man 15,65 mg Aminosäurestickstoff, nach Autoklavierung dagegen $17,34 \mathrm{mg}$, was besagt, daß nach dem Kochen über freier Flamme noch $9,7 \%$ peptidgebundenen Stickstoffs vorhanden sind. Ferner geht aus den ermittelten Zahlen hervor, daß Schwefelsäure bedeutend schwächer als Salzsäure wirkt, indem erst eine Säurestärke von 5-n-Schwefelsäure imstande ist, fast totale Hydrolyse $\mathrm{zu}$ erzeugen, während diese schon mit 3-n-HCl erzielt wird.

Um $\mathrm{zu}$ versuchen, ob es tunlich sei, eine Spaltung $\mathrm{zu}$ erzwingen, die stärker wäre als die durch 11/2stündige Autoklavierung auf $150^{\circ}$ erzeugte, verlängerten wir die Dauer der Autoklavierung von $1^{1 / 2}$ bis auf 3 Stunden; wie aber zu ersehen, nimmt der Abbau hierdurch nicht zu. Ferner unter- 
suchten wir, ob eine $1 \frac{1}{2}$ stündige stärkere Erhitzung (bis auf $180^{\circ}$ ) imstande war, die Menge formoltitrierbaren Stickstoffs zu vermehren. Die Zahlen für die Menge von Aminosäurestickstoff + Ammoniakstickstoff sind bei dieser starken Erhitzung fast dieselben wie bei Erhitzung auf $150^{\circ}$; da sich aber bei $180^{\circ}$ mehr Ammoniak bildet (abspaltet) als bei $150^{\circ}$, wird die Menge des Aminosäurestickstoffes bei $180^{\circ}$ geringer als bei $150^{\circ}$. Es scheint also, daß 11/2stündige Erhitzung auf $150^{\circ}$ die Erzielung des möglichst hohen Hydrolysegrades am meisten begünstigt.

Eine Versuchsreihe, die wir anstellten, um zu prüfen, ob die totale Hydrolyse des Hühnereiweißes durch 12 stündiges Kochen mit konzentrierter Salzsäure nicht zu ermöglichen sei, geben wir in untenstehender Tabelle III wieder.

\section{Tabelle III.}

Extraprobe: Lösung von trockenem Hühnereiweiß.

\begin{tabular}{|c|c|c|c|}
\hline \multicolumn{4}{|c|}{$\begin{array}{l}\text { Totalstickstoff }=28,00 \mathrm{mg} . \\
\mathrm{A}=\text { Ammoniakstickstoff }+ \text { Aminostickstoff } \\
\mathrm{B}=\text { Ammoniakstickstoff } \\
\mathrm{C}=\mathrm{A} \div \mathrm{B}=\text { Aminostickstoff. }\end{array}$} \\
\hline & A & B & C \\
\hline $\begin{array}{c}6 \text { Stunden mit } 20 \% \text { iger Salzsäure gekocht, } \\
\text { darauf eingedampft }\end{array}$ & 21,70 & 2,60 & 19,10 \\
\hline $\begin{array}{l}12 \text { Stunden mit } 20 \% \text { iger Salzsäure gekocht, } \\
\text { darauf eingedampft }\end{array}$ & 21,84 & 2,70 & 19,14 \\
\hline $\begin{array}{r}12 \text { Stunden mit } 5 \text {-normal-Schwefelsäure bei } 90^{\circ} \text {, } \\
\text { darauf } 6 \text { Stunden gekocht . . . }\end{array}$ & 21,56 & - & - \\
\hline $\begin{array}{c}12 \text { Stunden mit 5-normal-Schwefelsäure bei } 90^{\circ} \text {, } \\
\text { darauf } 12 \text { Stunden gekocht }\end{array}$ & 21,70 & - & - \\
\hline $\begin{array}{l}\text { Erhitzung im Autoklaven auf } 150^{\circ}-1^{1} / 2 \text { Stunden } \\
\text { mit 3-normal-Salzsäure }\end{array}$ & 23,10 & 2,70 & 20,40 \\
\hline $\begin{array}{l}\text { Erhitzung im Autoklaven auf } 150^{\circ}-1^{1 / 2} \text { Stunden } \\
\text { mit } 4 \text {-normal-Salzsäure } \ldots\end{array}$ & 23,24 & 2,75 & 20,49 \\
\hline $\begin{array}{c}\text { Erhitzung im Autoklaven auf } 150^{\circ}-11 / 2 \text { Stunden } \\
\text { mit 3-normal-Schwefelsäure }\end{array}$ & 22,40 & 2,90 & 19,50 \\
\hline
\end{tabular}

Die Zahlen zeigen ganz dasselbe Verhalten wie die der Tabelle II. Nach 12 stündigem Kochen mit starker Salzsäure findet man $19,14 \mathrm{mg} \mathrm{N}$, während $1^{1 / 2}$ stündige Behandlung im

Hoppe-Seyler's Zeitschrift f. physiol. Chemie. LXVII. 
Autoklaven mit 3-n-HCl bei $150^{\circ} 20,40 \mathrm{mg} \mathrm{N}$ gibt; es waren also nach Kochen über freier Flamme mit Salzsäure noch $6,2 \%$ peptidgebundenen Stickstoffs vorhanden. Ebenfalls zeigt der Versuch, daß 3-n- $\mathrm{H}_{2} \mathrm{SO}_{4}$ schwächer hydrolysierend wirkt als 3-n-HCl.

\section{Tabelle IV.}

Standardlösung $=$ ca. $4 \%$ ige alkalische Caseinlösung.

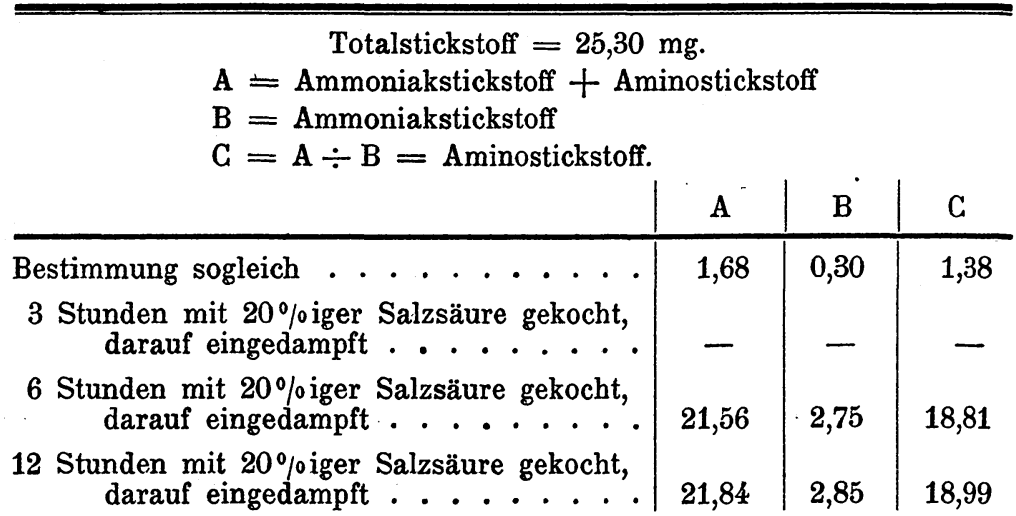

Spaltung durch Erhitzung im Autoklaven.

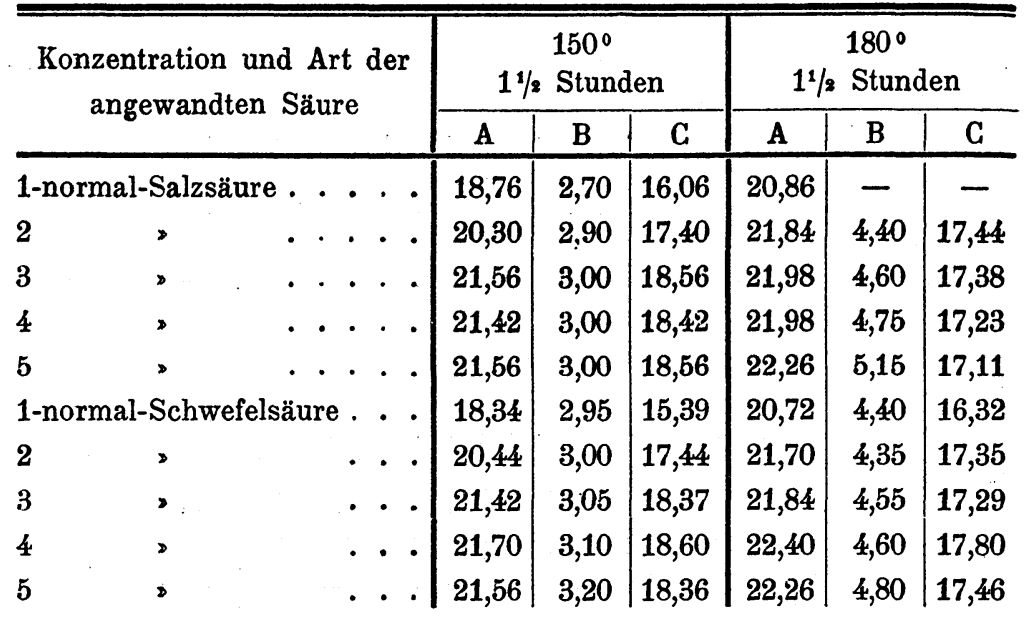

Die TabelleIV gibt die Resultate einer mit einer ca. $4 \%$ igen Caseinlösung angestellten Versuchsreihe. Es erweist sich, daß 6stündiges Kochen mit Salzsäure über freier Flamme genügt, 
um totale Hydrolyse hervorzurufen, indem die Menge des formoltitrierbaren Stickstoffes weder nach 12 stündigem Kochen noch nach Autoklavierung bei $150^{\circ}$ mit 3-n- $\mathrm{HCl}$ steigt. Ebenfalls zeigt es sich, daß schon $3-\mathrm{n}_{-} \mathrm{H}_{2} \mathrm{SO}_{4}$ eine fast totale Hydrolyse hervorzubringen vermag, was uns darlegt, daß Casein sich viel leichter als Hühnereiweiß hydrolysieren läßt.

Der Versuch zeigt ferner, daß Erhitzung im Autoklaven auf $180^{\circ} \mathrm{zwar}$ die Menge von Amino-N + Ammoniak-N vermehrt, daß die Menge des Aminosäurestickstoffes aber abnimmt, weil die starke Erhitzung bedeutend mehr Ammoniak abspaltet als die Erhitzung auf $150^{\circ}$.

Aus den Zahlen der Tabelle $\mathrm{V}$ geht hervor, daß eine Lösung von Witte-Pepton durch 12stündiges Kochen mit 20\% iger $\mathrm{HCl}$ oder mit 5-n-Schwefelsäure über freier Flamme vollständig gespalten wird. Nach $1^{1 / 2}$ stündigem Stehen im Autoklaven bei $150^{\circ}$ ist die Hydrolyse vollständig mit $2-n-H C l$ und mit 3-5-n- $\mathrm{H}_{2} \mathrm{SO}_{4}$. Erhitzt man 11/2 Stunden lang auf 180, so findet man eine Zunahme des Ammoniak- + Aminosäurestickstoffes; da die Ammoniakmenge aber stark gestiegen ist, wird die Menge des Aminosäurestickstoffes bei $180^{\circ}$ geringer als bei $150^{\circ}$.

Tabelle $\mathrm{V}$.

Ca. $4 \%$ ige Lösung von Witte-Pepton.

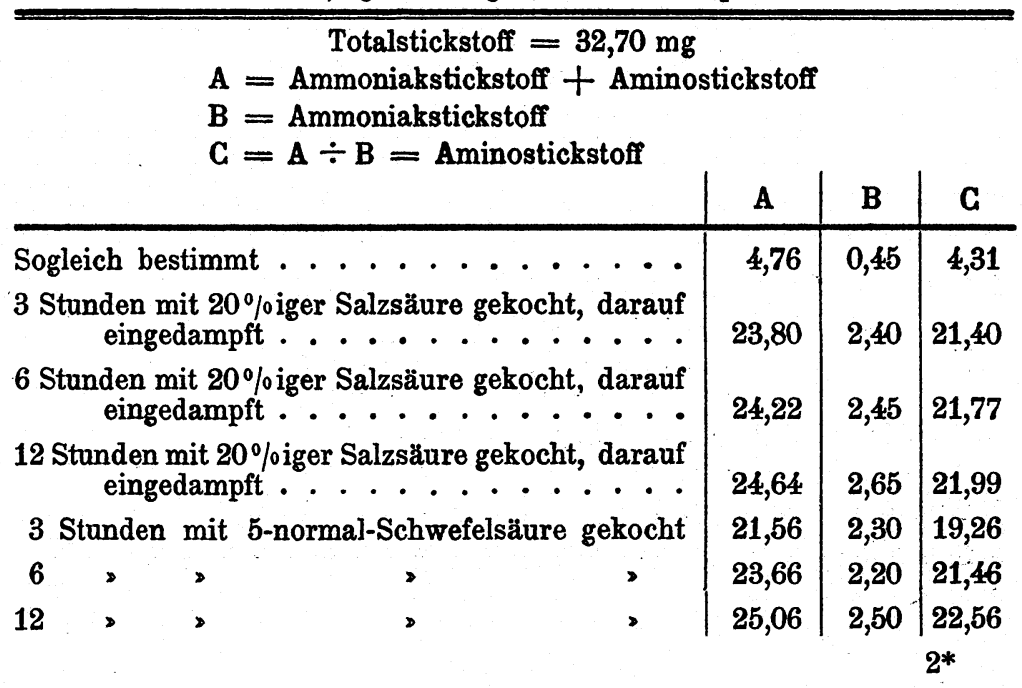


Spaltung durch Erhitzung im Autoklaven.

\begin{tabular}{|c|c|c|c|c|c|c|c|c|c|}
\hline \multirow{2}{*}{\multicolumn{4}{|c|}{$\begin{array}{l}\text { Konzentration und Art der } \\
\text { angewandten Säure }\end{array}$}} & \multicolumn{3}{|c|}{$\begin{array}{c}150^{\circ} \\
11 / 2 \text { Stunden }\end{array}$} & \multicolumn{3}{|c|}{$\begin{array}{c}180^{\circ} \\
1^{1 / 2} \text { Stunden }\end{array}$} \\
\hline & & & & A & B & C & A & B & C \\
\hline \multicolumn{2}{|c|}{ 1/2-normal-Salzsäure } & . . & - & 21,70 & 3,00 & 18,70 & 24,64 & 4,80 & 19,84 \\
\hline 1 & , & & & 24,36 & 3,20 & 21,16 & 25,48 & 4,60 & 20,88 \\
\hline 2 & , & $\cdot$ & ${ }^{\circ}$ & 25,48 & 3,10 & 22,38 & 26,04 & 4,80 & 21,24 \\
\hline 3 & $\gg$ & . & & 25,76 & 3,10 & 22,66 & 26,04 & 4,90 & 21,14 \\
\hline 4 & & 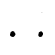 & 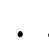 & 25,48 & 3,10 & 22,38 & 26,32 & 5,25 & 21,07 \\
\hline 5 & 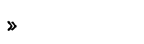 & & $e^{\circ}$ & 25,62 & 3,25 & 22,37 & 26,32 & 5,60 & 20,72 \\
\hline \multicolumn{3}{|c|}{ 1/2-normal-Schwefelsäure } & ?. & 16,94 & 2,65 & 14,29 & 22,40 & - & - \\
\hline 1 & , & & & 22,40 & 3,10 & 19,30 & 24,78 & - & - \\
\hline 2 . & > & & & 24,64 & 3,15 & 21,49 & 25,62 & 4,70 & 20,92 \\
\hline 3 & » & & . & 25,48 & 3,35 & 22,13 & 25,62 & 4,80 & 20,82 \\
\hline 4 & * & & . & 25,48 & 3,30 & 22,18 & 25,76 & 5,00 & 20,76 \\
\hline 5 & > & &. & 25,76 & 3,40 & 22,36 & 25,90 & 5,00 & 20,90 \\
\hline
\end{tabular}

Tabelle VI.

Phosphatfreie Lösung von verdautem Kalbfleisch V.

\begin{tabular}{|c|c|c|c|}
\hline \multicolumn{3}{|c|}{$\begin{array}{l}\quad \text { Totalstickstoff }=28,80 \mathrm{mg} \\
\mathrm{A}=\text { Ammoniakstickstoff }+ \text { Aminostickstoff } \\
\mathrm{B}=\text { Ammoniakstickstoff } \\
\mathrm{C}=\mathrm{A} \div \mathrm{B}=\text { Aminostickstoff }\end{array}$} & \multirow[b]{2}{*}{$\mathrm{C}$} \\
\hline & $\mathbf{A}$ & $\mathrm{B}$ & \\
\hline Sogleich bestimmt . . . . . . . . . . & 21,42 & 2,50 & 18,92 \\
\hline $\begin{array}{c}1 \text { Stunde mit } 20 \% \text { iger Salzsäure gekocht, darauf } \\
\text { eingedampft }\end{array}$ & 23,24 & 3,40 & 19,84 \\
\hline $\begin{array}{r}3 \text { Stunden mit } 20 \% \text { iger Salzsäure gekocht, darauf } \\
\text { eingedampft . . . . . . . . }\end{array}$ & 23,80 & 3,50 & 20,30 \\
\hline $\begin{array}{r}6 \text { Stunden mit } 20 \% \text { iger Salzsäure gekocht, darauf } \\
\text { eingedampft }\end{array}$ & 23,94 & 3,70 & 20,24 \\
\hline 1 Stunde mit 5-normal-Schwefelsäure gekocht. & 23,38 & 3,30 & 20,08 \\
\hline 3 Stunden & 23,66 & 3,30 & 20,36 \\
\hline 6. & 24,08 & 3,60 & 20,48 \\
\hline
\end{tabular}




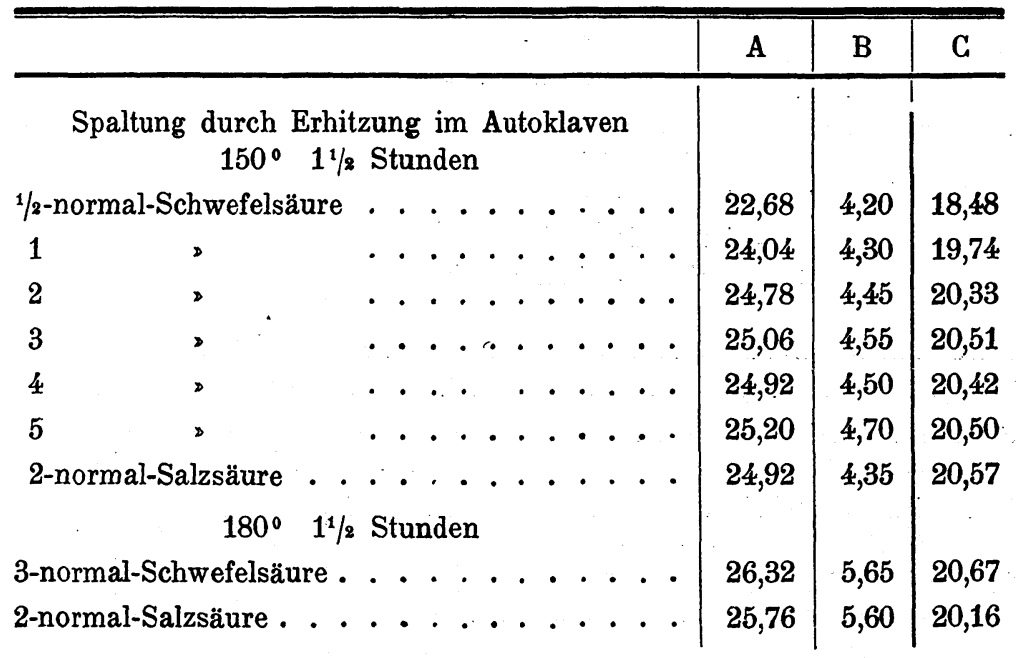

In den Tabellen VI und VII findet man die Ergebnisse von Spaltungsversuchen mit Fleisch, das längere Zeit (3 bis 4 Monate) hindurch mit Trypsin-Erepsin verdaut worden ist. Aús den Zahlen der Tab. VI sieht man, daß der hier angewandte Stoff vor der Behandlung mit Säure bei Formoltitrierung $18,92 \mathrm{mg}$ Stickstoff gab, während er nach $1^{1 / 2}$ stündiger $\mathrm{Be}$ handlung mit 2-n-Salysäure im Autoklaven bei $150^{\circ}$ ca $20,50 \mathrm{mg}$ formoltitrierbaren Stickstoff ergab, was mithin bezeichnet, daß der angewandte Stoff noch 7,7\% peptidgebundenen Stickstoffes enthielt.

Ferner sieht man aus der Tabelle, daß ein vollständiger Abbau schon nach 3stündigem Kochen mit 20\% oder 5-n-Schwefelsäure über freier Flamme, wie auch nach $1^{1 / 2}$ stündiger Autoklavierung auf $150^{\circ}$ mit 2-n-Schwefelsäure erreicht wird. Steigert man die Temperatur bei der Autoklavierung bis auf $180^{\circ}$, so findet man eine Zunahme der Ammoniakmenge, während die Menge des Aminosäurestickstoffs unverändert bleibt. 


\section{Tabelle VII.}

Phosphatfreie Lösung von verdautem Kalbfleisch.

\begin{tabular}{|c|c|c|c|c|c|c|}
\hline \multicolumn{7}{|c|}{$\begin{array}{l}\text { Totalstickstoff }=27,25 \mathrm{mg} \\
\mathrm{A}=\text { Ammoniakstickstoff }+ \text { Aminostickstoff } \\
\mathrm{B}=\text { Ammoniakstickstoff } \\
\mathrm{G}=\mathrm{A} \div \mathrm{B}=\text { Aminostickstoff }\end{array}$} \\
\hline & & & & $\mathbf{A}$ & B & C \\
\hline \multirow{3}{*}{\multicolumn{4}{|c|}{ 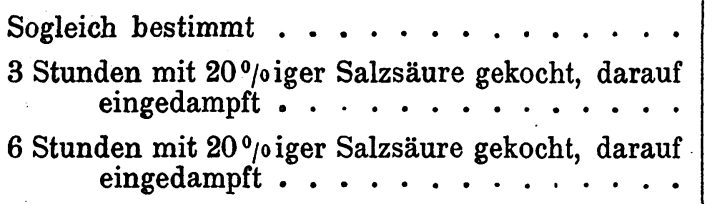 }} & & & \\
\hline & & & & 21,00 & 2,65 & 18,35 \\
\hline & & & & 21,84 & 2,75 & 19,09 \\
\hline \multirow{2}{*}{\multicolumn{7}{|c|}{$\begin{array}{r}12 \text { Stunden mit } 20 \% \text { iger Salzsäure gekocht, darauf } \\
\text { eingedampft } \\
\text { Spaltung durch Erhitzung im Autoklaven }\end{array}$}} \\
\hline & & & & & & \\
\hline \multirow{2}{*}{$\begin{array}{l}\text { Konzentration und Art der } \\
\text { angewandten Säure }\end{array}$} & \multicolumn{3}{|c|}{$11 / 2$ Stunden } & \multicolumn{3}{|c|}{$\begin{array}{ll}180^{\circ} \\
11 / 2 & \text { Stunden }\end{array}$} \\
\hline & $\mathbf{A}$ & B & C & $\bar{A}$ & B & C \\
\hline -normal-Salzsäure . & 22,12 & 3,35 & 18,77 & 23,10 & , 75 & \\
\hline . . & 22,40 & 3,30 & 19,10 & 23,52 & 4,80 & \\
\hline${ }^{\star}$ & 22,68 & 3,50 & 19,18 & 23,52 & 5,05 & 18,47 \\
\hline . . . & 22,54 & 3,60 & 18,94 & 23,52 & 5,10 & 18,42 \\
\hline . $\cdot$ & 22,54 & 3,60 & 18,94 & 23,80 & 5,40 & 18,40 \\
\hline -normal-Schwefelsäure . & 21,70 & 3,30 & 18,40 & 22,68 & 4,75 & 17,93 \\
\hline , & 22,40 & 3,40 & 19,00 & 23,24 & 4,90 & 18,34 \\
\hline . . . & 22,54 & 3,45 & 19,09 & 23,66 & 5,00 & 18,66 \\
\hline . . & 22,82 & 3,555 & 19,27 & 23,80 & 5,10 & 18,70 \\
\hline & 22,82 & 3,55 & 19,27 & 23,52 & 5,10 & 18,42 \\
\hline
\end{tabular}

Die Resultate der Tabelle VII, wo das angewandte verdaute Fleisch ca. 10\% peptidgebundenen Stickstoffs enthielt, sind ganz dieselben wie die der Tab. VI. Doch findet man hier bei Erhitzung auf $180^{\circ}$ geringere Werte des Aminosäurestickstoffs als bei $150^{\circ}$, indem zugleich die Ammoniakmenge bei $180^{\circ}$ um nicht so wenig höher ist als bei $150^{\circ}$. 
Tabelle VIII.

\begin{tabular}{|c|c|c|c|c|c|c|c|c|c|c|c|c|}
\hline \multirow{3}{*}{$\begin{array}{c}\text { Erhitzung } \\
\text { im } \\
\text { Autoklaven } \\
\text { mit } \\
\text { 3-normal- } \\
\text { Salzsäure }\end{array}$} & \multicolumn{12}{|c|}{$\begin{array}{l}\mathrm{A}=\text { Ammoniakstickstoff }+ \text { Aminostickstoff } \\
\mathrm{B}=\mathrm{Ammoniakstickstoff} \\
\mathrm{C}=\mathrm{A} \div \mathrm{B}=\text { Aminostickstoff }\end{array}$} \\
\hline & \multicolumn{3}{|c|}{$\begin{array}{c}\text { Lösung } \\
\text { von } \\
\text { trockenem } \\
\text { Hühnereiweiß } \\
\text { Total-N } \\
24,70 \mathrm{mg} \\
\end{array}$} & \multicolumn{3}{|c|}{$\begin{array}{c}\text { Alkalische } \\
\text { Caseinlösung } \\
\text { Total-N } \\
36,30 \mathrm{mg} \\
\end{array}$} & \multicolumn{3}{|c|}{\begin{tabular}{|c|} 
Lösung \\
von \\
Witte-Pepton \\
Total-N \\
$29,10 \mathrm{mg}$ \\
\end{tabular}} & \multicolumn{3}{|c|}{$\begin{array}{l}\text { Phosphatfreie } \\
\text { Lösung von } \\
\text { verdautem } \\
\text { Kalbfleisch V } \\
\text { Total-N } \\
29,70 \mathrm{mg}\end{array}$} \\
\hline & A & & C & A & & G & & B & C & A & D & \\
\hline Std. & & & 18,00 & 27,86 & $|3,65|$ & 24,21 & & $8|2,05|$ & & $|24,22|$ & {$[0, \pm 0$} & \\
\hline & 年 & & 16,92 & 27,30 & 3,60 & 23,70 & 20,58 & 8,00 & & 23,80 & {$[\tilde{e}, \pm 0 \mid$} & \\
\hline & & & $\mid 18,40$ & 20,10 & 0,00 & 24,90 & 22,54 & $x \mid<, 10$ & 20,4 & 24,64 & $\{0,0$ & 20 \\
\hline 3 & 20,58 & & 18,53 & 28,98 & 3,90 & 25,08 & 22,82 & 2,30 & & 24,64 & 3,9 & 20,6 \\
\hline $150^{\circ}, 1 / 2$ & 7,50 & & 10,1 & a & 3,55 & 22,77 & 21,00 & 0 & 18,95 & 24,08 & 3,45 & 20,00 \\
\hline 1 & , 30 & & $\mid 0,2 \mathrm{~V}$ & 20,20 & 3,90 & 25,08 & 22,68 & 82,30 & 20,38 & 24,78 & 4,00 & 20,78 \\
\hline & & & & & & & & & & & & \\
\hline
\end{tabular}

In der Tabelle VIII haben wir schließlich die Resultate einer Reihe von Versuchen angegeben, die wir anstellten; um zu untersuchen, welche Bedeutung Variationen des Erhitzungsgrades und der Erhitzungsdauer für den Abbau der Proteine und der nächsten Spaltungsprodukte derselben haben. Aus den gefundenen Zahlen sieht man, daß 3 stündige Erhitzung auf $120^{\circ}$ fast ganz dasselbe Resultat ergibt wie $1^{1 / 2}$ stündige Erhitzung auf $150^{\circ}$. Erhitzt man auf $135^{\circ}$, so wird 1 Stunde nicht zum völligen Abbau genügen; verlängert man aber die. Dauer bis auf 2 oder 3 Stunden, so erreicht man dasselbe Resultat wie durch $11 / 2$ stündige Erhitzung auf $150^{\circ}$.

Halbstündige Erhitzung auf $150^{\circ}$ bewirkt keinen vollständigen Abbau, dagegen gibt die 1stündige dasselbe Resultat wie die $1^{1 / 2} / 2$ stündige Erhitzung.

Wie oben angegeben, bestimmt man die Menge des Aminosäurestickstoffes durch die Differenz zwischen Aminosäurestickstoff + Ammoniakstickstoff (der durch die Formoltitrierung bestimmt wird) und dem Ammoniakstickstoff, der durch Destil- 
lation im Vakuum mit Baryt in Methylalkohol bestimmt wird. Beim Durchsehen der verschiedenen Versuche findet man indes, daß die Ammoniakmenge, was eine und dieselbe Lösung betrifft, je nach den verschiedenen Einwirkungen, denen die Lösung ausgesetzt wurde, stark variieren kann. Als Beispiel des hier Angeführten können wir der Tab. V einige Zahlen entlehnen; zu diesem Versuche wurde eine Lösung von Witte-Pepton mit einem Totalstickstoffgehalt (in $5 \mathrm{ccm}$ ) $=32,70 \mathrm{mg}$ angewandt. Die Zahlen stellen sich wie folgt:

$$
\begin{aligned}
& \mathrm{A}=\text { Amino- }+ \text { Ammoniakstickstoff } \\
& \mathrm{B}=\text { Ammoniakstickstoff } \\
& \mathrm{C}=\mathrm{A} \div \mathrm{B}=\text { Aminostickstoff. }
\end{aligned}
$$

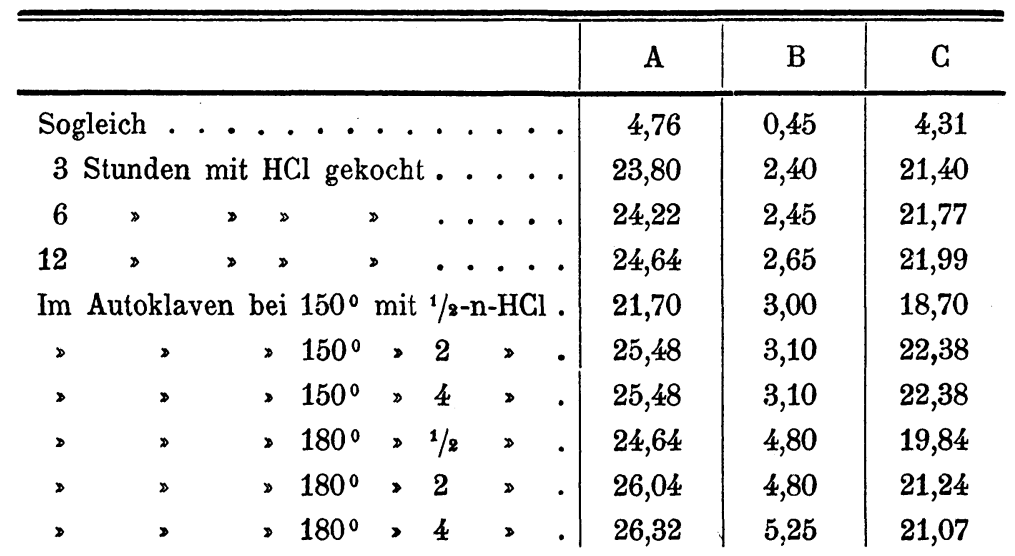

Betrachten wir die oben angeführten Zahlen, so finden wir, daß die Ammoniakabspaltung beim Kochen über freier Flamme am geringsten ist, so zwar, daß die Ammoniakmenge immer mehr zunimmt, je länger das Kochen dauert. Bei Autoklavierung auf $150^{\circ}$ ist die Ammoniakabspaltung stärker als beim Kochen über freier Flamme, und bei $180^{\circ}$ ist die Ammoniakmenge noch ferner sogar ziemlich bedeutend gestiegen. Betrachten wir darauf die Zahlen für Aminosäurestickstoff + Ammoniakstickstoff (Kolonne A), so sehen wir, daß das Kochen über freier Flamme niedrigere Zahlen gibt als das Erhitzen auf $150^{\circ}$ und daß die höchsten Zahlen durch Erhitzung auf $180^{\circ}$ erzielt werden. Da jedoch das Steigen der Zahlen der 
Kolonne A nicht mit dem Steigen der Menge abgespaltenen Ammoniaks zusammengeht, so ergiebt sich das Resultat, daß die Menge des Aminosäurestickstoffes (Kolonne C) bei Erhitzung auf $150^{\circ}$ und beim Kochen mit HCl über freier Flamme um nicht so gar wenig höher wird als bei Erhitzung auf $180^{\circ}$. Den Grund dieses eigentümlichen Verhaltens hat man gewiß in dem Umstand zu suchen, daß gleichzeitig mit dem Abbau der Proteine eine sekundäre Spaltung der gebildeten Aminosäuren stattfindet, mittels deren Ammoniak abgespalten wird. ${ }^{1}$ ) Das Resultat dieser beiden gleichzeitig verlaufenden Prozesse wird natürlich in erster Linie von der Art der sekundär gespaltenen Aminosäuren abhängig sein. Wenn z. B. Glycin oder andere Monoaminosäuren sämtlichen Stickstoff als Ammoniak abspalten, so wird das Sinken des Aminosäurestickstoffes gleich dem Steigen des Ammoniakstickstoffes werden. Handelt es sich um Stoffe wie z. B. Tryptophan, so wird bei vollständiger Abspaltung das Sinken des Aminosäurestickstoffes geringer sein als das Steigen des Ammoniakstickstoffes. Wird dagegen Arginin durch Zerteilung des Guanidins gespalten, so erhalten wir gleichzeitig mit einer Zunahme der Ammoniakmenge auch ein Steigen der Aminosäuremenge; dieses Verhalten wird jedoch, wie genannt, nicht eintreten, wenn man dafür sorgt, daß man bei Temperaturen bleibt, die $150^{\circ}$ nicht übersteigen.

Es läßt sich also nicht mit Sicherheit sagen, wann der Abbau eines Proteinstoffes vollständig ist. Indes glauben wir,

1) Diese Abspaltung erfolgt nicht mit bezug auf alle Aminosäuren gleich leicht. So spaltet Glycin bei Erhitzung mit 2-n- $\mathrm{HCl}$ auf $150^{\circ}$ gar kein Ammoniak ab. Eine Tyrosinlossung mit einem Totalstickstoffgehalt von $19,00 \mathrm{mg}$ gab nach derselben Behandlung nur 0,2 mg Ammoniak- $\mathrm{N}$ und $0,2245 \mathrm{~g}$ Histidin $(60,84 \mathrm{mg} \mathrm{N}$ entsprechend) nur $0,3 \mathrm{mg}$ Ammoniak- $\mathrm{N}$. Dagegen ergaben $0,3072 \mathrm{~g}$ Cystin $(35,84 \mathrm{mg} \mathrm{N}$ entsprechend) nach Autoklavierung mit 2-n-HCl 11,0 mg Ammoniak-N. Mit Bezug auf das Arginin gaben Schulze und Winterstein (Diese Zeitschrift, Bd. XXXIV) an, dieser Stoff ertrage 10 stündiges Kochen mit konzentrierter Salzsäure, ohne daß sich Ammoniak abspalte; erhitze man das Arginin mit konz entrierter Salzsäure in zugeschmolzenen Glasröhren bis auf $150^{\circ}$, so sei die Ammoniakbildung nur gering und erst bei 180-200 erfolge eine stärkere Abspaltung des Ammoniaks. 
daß der in der Einleitung dieser Abhandlung erwähnte "Nullpunkt» dort zu suchen ist, wo wir bei einer verhältnismäßig geringen Ammoniakbildung den höchsten Wert der Menge des Aminosäurestickstoffs erhalten, und unter Berücksichtigung der oben angeführten Versuche muß man annehmen, daß dieser Nullpunkt höchst wahrscheinlich durch $11 / 2$ stündige Erhitzung im Autoklaven auf $150^{\circ}$ mit 3-n-HCl erreicht wird.

Aus dem Obenstehenden geht hervor, daß wir imstande sind, mittels der Sörensenschen Formoltitrierung zu entscheiden, ob der Abbau eines Proteins durch Einwirkung von z. B. Säuren oder Fermenten vollständig ist oder nicht, und wenn der Abbau nicht vollständig ist, dann zu entscheiden; wieviel peptidgebundener Stickstoff sich noch im untersuchten Stoffe befindet. Obschon Sörensens erste Mitteilung bereits 1907 veröffentlicht wurde, hat die Formoltitrierung merkwürdigerweise nur sehr geringe Anwendung gefunden; dies muß um so mehr Erstaunen erregen, als das Verfahren sehr leicht auszuführen ist und die bisher angewandten Verfahrungsarten an Genauigkeit weit übertrifft.

Namentlich Abderhalden und seine Mitarbeiter haben während der letzteren Jahre eine sehr bedeutende Arbeit ausgeführt, um zu entscheiden, ob Proteine, die 3-4 Monate lang mit Trypsin + Erepsin verdaut wurden, wirklich als vollständig abgebaut zu betrachten seien. Die genannten Forscher scheinen anzunehmen, daß Fermente wirklich imstande seien, die totale Hydrolyse von z. B. Casein oder Fleisch zu bewirken. Wir hatten in der jüngsten Zeit die Gelegenheit, eine Reihe Präparate aus trypsin-erepsin-verdautem Fleische zu untersuchen, und haben gefunden, daß diese Präparate ca. 6-12\% peptidgebundenen Stickstoffs enthalten. Es unterliegt wohl keinem Zweifel, daß so kleine Mengen sich mittels der von Abderhalden und seinen Mitarbeitern angewandten Methode nicht nachweisen lassen; erstens ist die quantitative Bestimmung der einzelnen Aminosäuren mit ziemlich großen Fehlern behaftet und zweitens findet sich in den vollständig abgebauten Proteinen eine ziemlich bedeutende Menge unbekannter Stoffe. 
Wenn man deshalb, wie Abderhalden und seine Mitarbeiter, zu Fütterungsversuchen trypsin-erepsin-verdaute Proteine benutzt und aus den Versuchen Folgerungen in betreff der Bedeutung «total» abgebauter Proteine für den Stoffwechsel zieht, so ist dies nicht ganz berechtigt; wir müssen im Interesse der ganzen Forschung jedenfalls verlangen, über den Hydrolysegrad der angewandten Proteine genau orientiert zu werden. Unserer Auffassung zufolge kann das nur durch Anwendung der Formoltitrierung geschehen, die sich in der oben angegebenen Weise verhältnismäßig leicht durchführen läßt. 